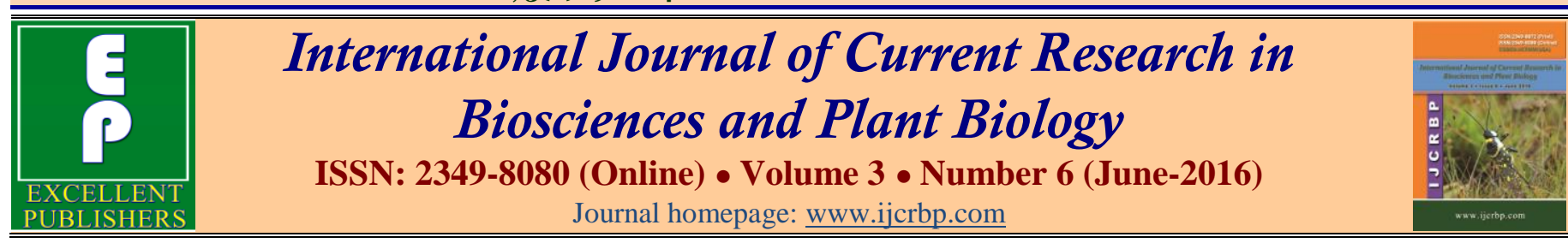

\title{
A Study on the Plant Biocultural Diversity of Palrampattu and Vadakanandal Sacred Groves in Villupuram District, Tamil Nadu
}

\author{
M. Subramanian1* ${ }^{*}$ S. Karthik¹, S. Ravikumar² and R. Dhamodaran3 \\ ${ }^{1}$ Ph.D. Scholars, P.G. and Research Department of Plant Biology and Plant Biotechnology, Presidency College (Autonomous), \\ Kamarajar Road, Chennai-6oo oo5, Tamil Nadu, India \\ ${ }^{2}$ Assistant Professor, P.G. and Research Department of Plant Biology and Plant Biotechnology, Presidency College \\ (Autonomous), Kamarajar Road, Chennai 6oo oo5, Tamil Nadu, India \\ 3 Associate Professor, P.G. and Research Department of Plant Biology and Plant Biotechnology, Presidency College \\ (Autonomous), Kamarajar Road, Chennai 600 oo5, Tamil Nadu, India \\ *Corresponding author.
}

\begin{tabular}{|c|c|}
\hline Abstract & Article Info \\
\hline $\begin{array}{l}\text { Floristic diversity associated with the cultural activities of people in Palrampattu and } \\
\text { Vadakanandal sacred groves of Villupuram district, Tamil Nadu, India were explored in } \\
\text { the present study. Mimosaceae ( } 11 \text { species), Caesalpiniaceae ( } 10 \text { species) were the } \\
\text { dominant families in the Palrampattu grove followed by Fabaceae (9 species), } \\
\text { Apocynaceae ( } 8 \text { species), Acanthaceae ( } 7 \text { species), Malvaceae ( } 7 \text { species), Asteraceae ( } 6 \\
\text { species), Capparaceae ( } 6 \text { species), Rubiaceae ( } 6 \text { species), Euphorbiaceae ( } 5 \text { species), } \\
\text { Rutaceae ( } 5 \text { species). Mimosaceae ( } 12 \text { species), Fabaceae ( } 10 \text { species) were the } \\
\text { dominant families in Vadakanandal sacred grove. Culturally, the deities associated with } \\
\text { these groves are: Aagasa Karuppusami, Periyakruppu and Nondikaruppu. A total of } 190 \\
\text { plant species belonging to } 168 \text { genera were recorded from the Palrampattu sacred grove } \\
\text { which included herbs ( } 59 \text { species), shrubs ( } 28 \text { species), climbers ( } 43 \text { species), trees ( } 58 \\
\text { species), and parasite ( } 2 \text { species). In Vadakanandal sacred grove, a total of } 178 \text { species } \\
\text { belonging to } 159 \text { genera consisting of herbs }(54 \text { species), shrubs ( } 27 \text { species), climbers } \\
\text { (39 species), trees ( } 56 \text { species) and parasites ( } 2 \text { species) were reported. Sacred groves } \\
\text { are one of the most valuable sources of plant diversity and ethnobotany, non-timber } \\
\text { forest products and cultural ethos. In short, the floristic diversity of the Palrampattu and } \\
\text { Vadakandhal sacred groves act as a storehouse of medicinal plants. }\end{array}$ & $\begin{array}{l}\text { Accepted: } 23 \text { May } 2016 \\
\text { Available Online: 06 June } 2016 \\
\text { K e y w o r d s } \\
\text { Cultural beliefs } \\
\text { Palrampattu } \\
\text { Plant diversity } \\
\text { Sacred groves } \\
\text { Vadakanandal }\end{array}$ \\
\hline
\end{tabular}

\section{Introduction}

Sacred groves may be defined as pristine patches of native biodiversity that are persevered in their original state over the centuries. This is mainly due to the religious beliefs, taboos and customs that have been handed down from generation to generation. They are distinct segments of various landscapes containing trees and other life forms and geographical features (Gadgil and Vartak, 1975). Gadgil's group at the Indian Institute of Science in Bangalore first reported the existence of a pristine grove in the Western Ghats. The cultural links to conservation was the central theme whereas the ecological and biological values were highlighted by Ramakrishnan (1998), Malhotra et al. (2001) and Ramanujam et al. (2002) which were concise but comprehensive too. Indian society is thus an agglomeration of about 40,000 endogamous groups of castes and communities including 3000 tribal sects (Malhotra, 2001). This diversity is reflected in the variety of cultures which integrated the concept of sacred groves in their own way and practiced with characteristic 
traditions, rites and rituals. Rightly, this is called 'vernacular conservation'. Soon they found hundreds of such groves on the Western Ghats in the States of Maharashtra and Karnataka (Gadgil and Vartak, 1981). In Southern India, groves have been reported by different groups from Uttara and Dakshina Kannada regions, from southern and northern Kerala, and from Tamil Nadu.

\section{Sacred groves - an overview}

Historically, the sacred groves could be traced back to the hunter- gatherer societies which viewed the environment as an inalienable part of their life. Such societies consisted of "ecosystem people" as they were truly conscious of their total dependence on nature and natural resources, and hence worshipped nature as God. Sooner or later, when population increased they cleared large tracts of forests. Fortunately, the religions that subsequently invaded the societies also approved of and practiced the sacred grove concept. From strictly including only the climax forest patches (Gadgil and Vartak, 1981), two types - village groves near the hamlets and far off sacred groves were recognized. 'Aswathakattes' are paired trees of neem and pipal while Tanchavati' is a group of five species of trees; 'Banni Mantaps' are clusters of Acacia ferruginia trees; rarely a large banyan tree may represent the grove as well (Somasekhar, 1998). Each ecosystem had people carrying out their unique habits of hunting, gathering, cultivating and worshipping deities. Although some of the deities may not be associated with extensive forest cover, most are found intimately connected with at least a small grove of plants. Each grove is dedicated to the local folk deities and spirits (vanadevathai) and has folklore associated with either the deity or the grove. Commonly found deities are Aiyanar, Sastha, Muniyappa, Karuppuswami, Veeran (Kaaval Teivam / protective deity), Andavar (a powerful wish-fulfilling deity) and the goddesses are Selliyamman, Kali, Ellaikali, Ellaipidari, Sapta Kannis, Pechiyamman, Rakkachiyamman and Nagadevadhai (fertility and good health). In certain sacred groves, people fulfill their vows by tonsuring (shaving the head to make a ceremonial offering of hair to the god). Terracotta horses of various sizes are lined up in front of the deity within the sacred groves praying for a good harvest (Kadamban, 1998).

Sacred groves were first described in India by Dietrich Brandis in 1857. According to Fergusson (1971), sacred groves are believed to be pre-Vedic in origin. More recently, Gadgil and Vartak (1981) reviewed the sacred groves of India's states and reported some 13,270 intact sacred groves, with the highest number found in Himachal Pradesh (nearly 5000 sacred groves). Other estimates put the total number of such groves in India at over a lakh. In culturally vibrant Tamil Nadu, over 1200 sacred groves have been reported (Amirthalingam, 2012). Maheswaran et al. (1995) studied the floristic of a miniature sacred grove measuring the size of a basket ball court on the Passumari hill-top near Vedanthangal bird sanctuary in Kanchipuram district of Tamil Nadu. Generally, the sacred groves of Tamil Nadu are dedicated to Aiyanar and/or Amman. Footwear or any other leather wear is not permitted inside the grove. In certain cases, people observe 'viradh' (fasting) prior to ceremonial visits to the grove (http://www.ecoheritage. cpreec.org)

\section{Biodiversity and vegetation types}

The compositions of the vegetation of the groves are considered climax vegetation of the respective areas (Gadgil and Vartak, 1981). The World Conservation Strategy (WCS) has appreciated the ecological prudence imbued in the concept of sacred groves and recommended that the cultural connections to conservation practices as prevailing in the classical societies be encouraged. Such an attempt would also directly involve the local people in biodiversity conservation. Thus it emerges from the foregoing that the status report of sacred groves in India is still incomplete despite an intensive survey; the analysis of the floristic and phyto-sociology of the selected grove are still ongoing. Thirdly, the aim is to document in detail the belief systems, rites and rituals, folklore and their impact on biodiversity conservation and finally, to make a comprehensive inventory of the species covering the Palrampattu and Vadakanandal groves and to assess the conservation value to bring out the comprehensive plant biodiversity of the sacred groves in Kallakurichi taluk.

\section{Materials and methods}

This study envisages the estimation of floral wealth of the sacred groves in Palrampattu (Fig. 1) and Vadakanandal (Fig. 2), understanding the vegetation from a holistic point of view and ascertaining their floristic composition (Fig. 3). First hand information about the existence of sacred groves was gathered from hereditary priests and the temple authorities, environmental action groups and various social organizations. With this background information, 
thorough field surveys were carried out by visiting the sacred grove and the neighbouring people were interviewed to confirm the information about the presence of other groves in the vicinity. For this study, the Palrampattu and Vadakanandal grove has taken for detailed analysis. The data collection was done from these sacred groves through field survey. Plants either with flowers or fruits were collected and identified or confirmed with available regional floras of Gamble (1915 - 1936), Matthew (1982, 1983 and 1988), Nair and Henry (1983), Henry et al. (1987, 1989), Sanjappa (1992), Balakrishnan and Chakrabarthy (2007), and Karthikeyan et al. (2009). Photographic documentation has also been done. Herbaria were prepared for all the plants and deposited in the Presidency College, Chennai.

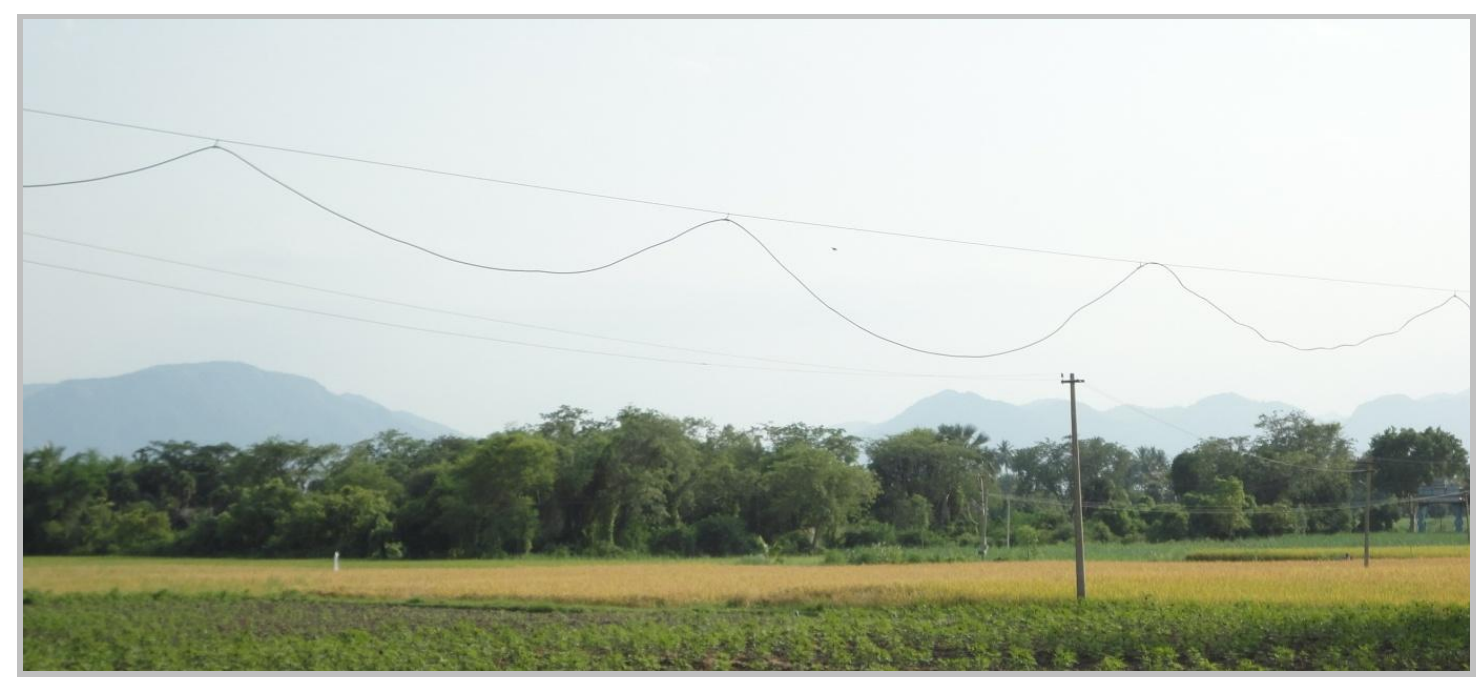

Fig. 1: An outer view of Palrampattu sacred grove.

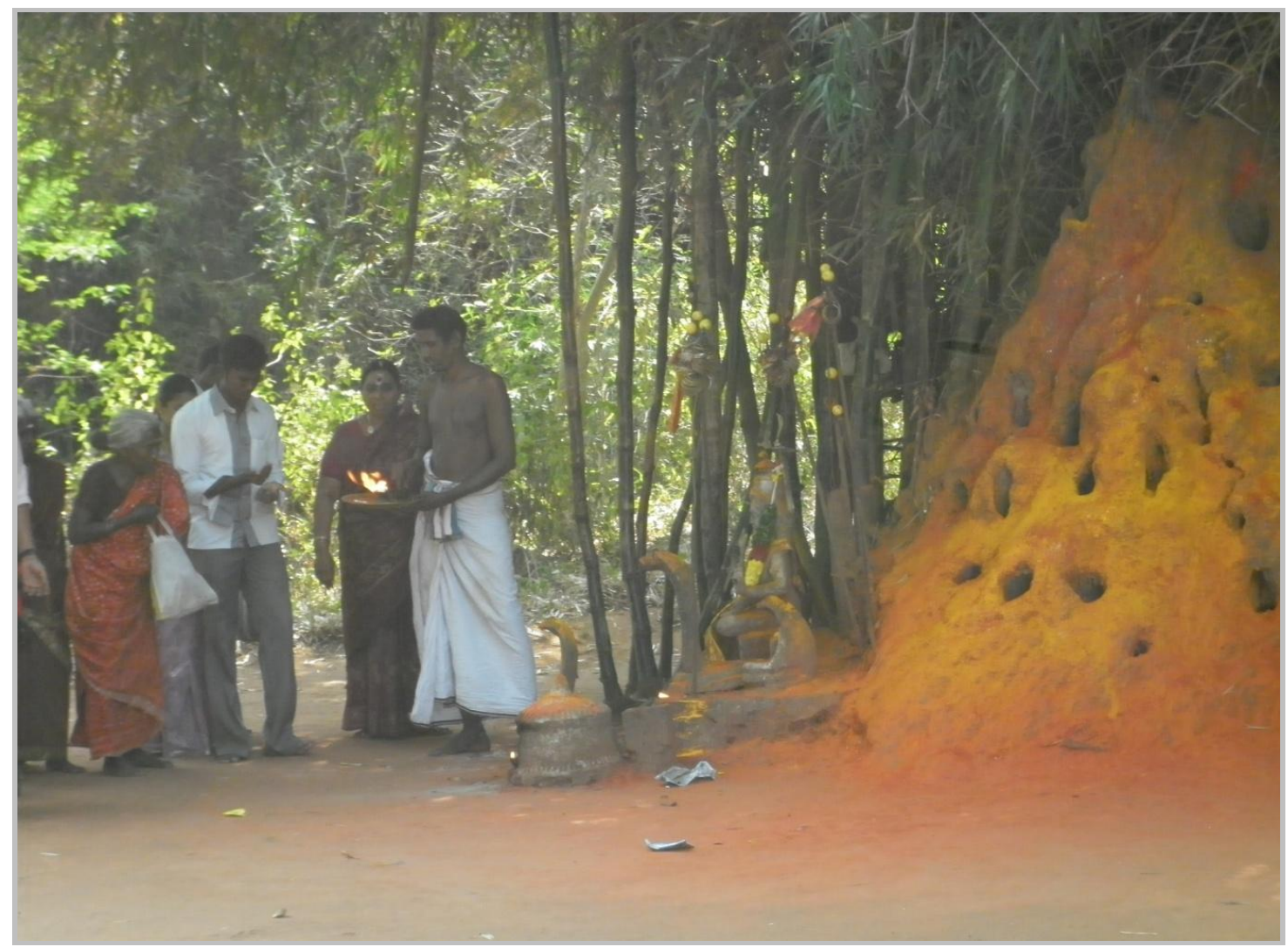

Fig. 2: Deities in Vadakanandal sacred grove. 


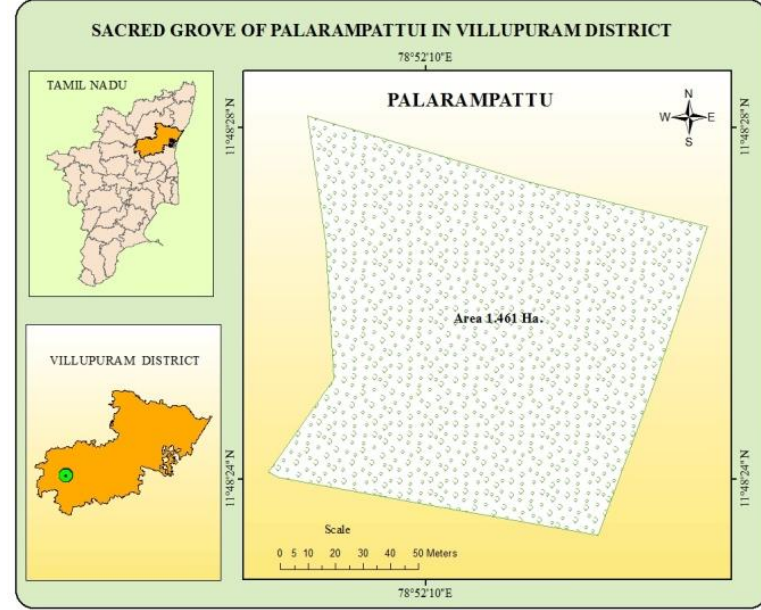

Fig. 3: Location of the study site.

\section{Results and discussion}

Floristic analysis of Palrampattu has been defined as the theoretical study of classification including its basis, principal, procedures and rules. The results of the pooled data of semi-evergreen vegetation type indicate the species richness of the grove. There are 190 species recorded in Palrampattu sacred grove (Table 1 and Fig. 4) and 178 species in Vadakanandal grove (Table 2 and Fig. 5). The present observation is very close to the range of plant species recorded by Karthik et al. (2015) who reported a total of 185 plant species belonging to 158 genera and 58 families in Kilcheruvi (Edaicheruvi) sacred grove of Cuddalore district, south India. In the present study, Mimosaceae (11 species), Caesalpiniaceae (10 species) were the dominant families followed by Fabaceae (9 species), Apocynaceae (8 species), Acanthaceae (7 species), Malvaceae (7 species), Asteraceae (6 species), Capparaceae (6 species), Rubiaceae (6 species), Euphorbiaceae (5 species), Rutaceae (5 species) in Palrampattu sacred grove. Amaranthaceae, Arecaceae, Asparagaceae, Cactaceae, Celastraceae, Ebenaceae, Loganiaceae, Molluginaceae and Myrtaceae were represented by single species each in Palrampattu grove. Mimosaceae (12 species), Fabaceae (10 species) were found to be the dominant families in the Vadakanandal sacred grove followed by Apocynaceae ( 9 species), Caesalpiniaceae (8 species), Acanthaceae (7 species), Capparaceae (6 species), Rubiaceae (6 species). The families which showed minimum number of species in Vadakanandal sacred grove were: Alangiaceae, Anacardiaceae, Annonaceae, Araceae, Aristolochiaceae, Asphodelaceae, Bignoniaceae, Cactaceae, Casuarinaceae, Commelinaceae, Commelinaceae, Commelinaceae,

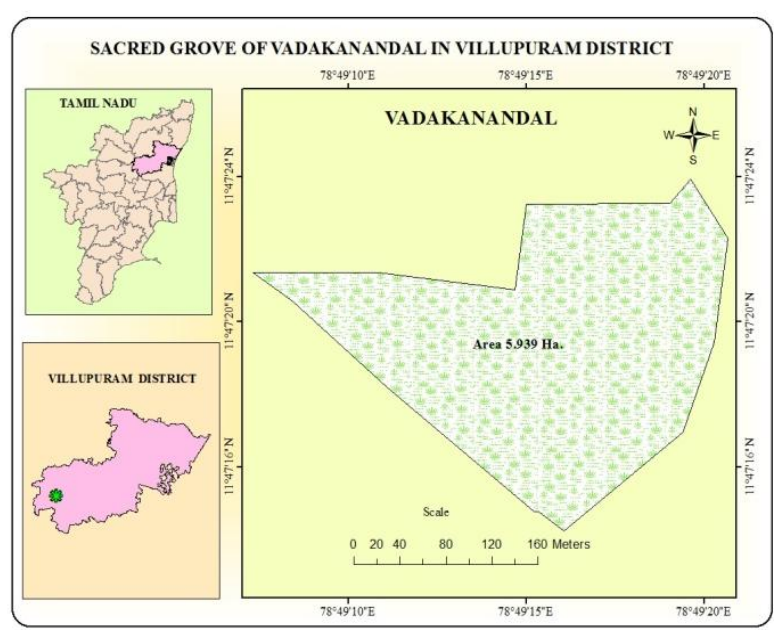

Ebenaceae, Flacourtiaceae, Hernandiaceae, Lauraceae, Loranthaceae, Meliaceae, Moringaceae, Myrtaceae, Passifloraceae, Pedaliaceae, Plumbaginaceae, Sapotaceae, Scrophulariaceae, Violaceae, Vitaceae and Zygophyllaceae.

\section{Cultural ethos of the groves}

In addition to restrictive practices, people observe rituals and organize festivals to please the supernatural forces for warding off the dangers in the form of drought, sickness, epidemics etc and for seeking a rich harvest and good health. Offerings are made naturally during festivals and these include miniatures of horses, bulls and elephants, the last one being characteristic of the coastal groves; the terracotta images were smaller in olden days but have assumed gigantic proportions recently (Amirthalingam, 1998). The philosophical underpinning in the making of a terracotta image is interesting. Parts of the old terracotta images or soil from the grove are pounded and mixed with clay for making new ones. The cyclic role of clay is correlated to the Hindu philosophy of birth, death and rebirth. The images of horses, elephants, or bulls represent the finiteness of a human life time. Customarily, terracotta images are offered, but figures from lime - stone or granite were also offered in earlier days. In the agricultural plains of Tamil Nadu, such events are part of the annual village festival during which a day is allotted for Aiyanar grove. The rites and ritual prevailing in the Puducherry region have been documented recently (Ramanujam et al., 2002). People perform important domestic ceremonies like tonsuring or ear-boring of the first born-child in the family. They also customarily offer invitations for their domestic functions like marriages. 
Table 1. List of plants recorded in the Palrampattu sacred grove.

\begin{tabular}{|c|c|c|c|}
\hline S. No. & Botanical name & Habit & Family \\
\hline 1 & Abrus precatorius L. & $\mathrm{C}$ & Fabaceae \\
\hline 2 & Abutilon indicum (L.) Sweet & $\mathrm{H}$ & Malvaceae \\
\hline 3 & Acacia ferruginea DC. & $\mathrm{T}$ & Mimosaceae \\
\hline 4 & Acacia leucophloea (Roxb.) Willd. & $\mathrm{T}$ & Mimosaceae \\
\hline 5 & Acacia caesia $(\mathrm{L}$.$) Willd.$ & $\mathrm{C}$ & Mimosaceae \\
\hline 6 & Acacia nilotica (L.) Delile & $\mathrm{T}$ & Mimosaceae \\
\hline 7 & Acalypha indica L. & $\mathrm{H}$ & Euphorbiaceae \\
\hline 8 & Aerva lanata (L.) Juss. ex Schult. & $\mathrm{H}$ & Amaranthaceae \\
\hline 9 & Alangium salviifolium (L. f.) Wangerin & $\mathrm{T}$ & Alangiaceae \\
\hline 10 & Albizia amara (Roxb.) Boivin & $\mathrm{T}$ & Mimosaceae \\
\hline 11 & Albizia lebbeck (L.) Benth. & $\mathrm{T}$ & Mimosaceae \\
\hline 12 & Aloe vera (L.) Burm.f. & $\mathrm{H}$ & Asphodelaceae \\
\hline 13 & Amarantus viridis L. & $\mathrm{H}$ & Amaranthaceae \\
\hline 14 & Andrographis paniculata (Burm.f.) Nees & $\mathrm{H}$ & Acanthaceae \\
\hline 15 & Annona squamosa $\mathrm{L}$. & $\mathrm{T}$ & Annonaceae \\
\hline 16 & Apluda mutica $\mathrm{L}$. & $\mathrm{H}$ & Poaceae \\
\hline 17 & Asparagus racemosus Willd. & $\mathrm{C}$ & Asparagaceae \\
\hline 18 & Asystasia gangetica (L.) T.Anderson & $\mathrm{H}$ & Acanthaceae \\
\hline 19 & Atalantia monophylla (L.) DC. & $\mathrm{T}$ & Rutaceae \\
\hline 20 & Azadirachta indca a. Juss. & $\mathrm{T}$ & Meliaceae \\
\hline 21 & Azima tetracantha Lam. & $\mathrm{S}$ & Salvadoraceae \\
\hline 22 & Bambusa bambos (L.) Voss & $\mathrm{T}$ & Poaceae \\
\hline 23 & Barleria prionitis L. & $\mathrm{H}$ & Acanthaceae \\
\hline 24 & Bauhinia racemosa Lam. & $\mathrm{T}$ & Caesalpiniaceae \\
\hline 25 & Blepharis maderaspatensis (L.) B.Heyne ex Roth & $\mathrm{H}$ & Acanthaceae \\
\hline 26 & Blumea obliqua (L.) Druce & $\mathrm{H}$ & Asteraceae \\
\hline 27 & Boerhavia diffusa $\mathrm{L}$. & $\mathrm{H}$ & Nyctaginaceae \\
\hline 28 & Borassus flabellifer $\mathrm{L}$. & $\mathrm{T}$ & Arecaceae \\
\hline 29 & Bougainvillaea spectabilis, Willd. & $\mathrm{C}$ & Nyctaginaceae \\
\hline 30 & Butea monosperma (Lam.) Taub. & $\mathrm{T}$ & Fabaceae \\
\hline 31 & Cadaba fruticosa (L.) Druce & $\mathrm{S}$ & Capparaceae \\
\hline 32 & Caesalpinia bonduc (L.) Roxb. & $\mathrm{C}$ & Caesalpiniaceae \\
\hline 33 & Calotropis gigantea (L.) Dryand. & $\mathrm{S}$ & Asclepiadaceae \\
\hline 34 & Canavalia virosa (Roxb.) & $\mathrm{C}$ & Fabaceae \\
\hline 35 & Cansjera rheedii Gmel. & $\mathrm{C}$ & Opiliaceae \\
\hline 36 & Canthium coromandelicum (Burm.f.) Alston & $\mathrm{S}$ & Rubiaceae \\
\hline 37 & Capparis sepiaria L. & $\mathrm{C}$ & Capparaceae \\
\hline 38 & Capparis zeylanica $\mathrm{L}$. & $\mathrm{C}$ & Capparaceae \\
\hline 39 & Cardiospermum helicacabum L. & $\mathrm{C}$ & Sapindaceae \\
\hline 40 & Carissa spinarum $\mathrm{L}$. & $\mathrm{S}$ & Apocynaceae \\
\hline 41 & Cascabela thevitia (L.) Lipp. & $\mathrm{T}$ & Apocynaceae \\
\hline 42 & Cassia fistula $\mathrm{L}$. & $\mathrm{T}$ & Caesalpiniaceae \\
\hline 43 & Cassine glauca (Rottb.) Kuntze & $\mathrm{T}$ & Celastraceae \\
\hline 44 & Cassytha filiformis $\mathrm{L}$. & $\mathrm{P}$ & Lauraceae \\
\hline 45 & Catharanthus roseus (L.) G.Don & $\mathrm{H}$ & Apocynaceae \\
\hline 46 & Catunaregam spinosa (Thunb.) Tirveng & $\mathrm{T}$ & Rubiaceae \\
\hline 47 & Cereus pterogonus Lam. & $\mathrm{S}$ & Cactaceae \\
\hline 48 & Chloris barbata $\mathrm{Sw}$. & $\mathrm{H}$ & Poaceae \\
\hline 49 & Chloroxylon swietenia DC. & $\mathrm{T}$ & Rutaceae \\
\hline 50 & Cissampelos pareira $\mathrm{L}$. & $\mathrm{C}$ & Menispermaceae \\
\hline 51 & Cissus quadrangularis L. & $\mathrm{C}$ & Vitaceae \\
\hline 52 & Citrus aurantiifolia (Christm.) Swingle & $\mathrm{T}$ & Rutaceae \\
\hline 53 & Cleome gynandra L. & $\mathrm{H}$ & Capparaceae \\
\hline 54 & Cleome viscosa $\mathrm{L}$. & $\mathrm{H}$ & Capparaceae \\
\hline 55 & Clerodendrum phlomidis L. f. & $\mathrm{H}$ & Laminaceae \\
\hline
\end{tabular}




\begin{tabular}{|c|c|c|c|}
\hline S. No. & Botanical name & Habit & Family \\
\hline 56 & Clitoria ternatea $\mathrm{L}$. & $\mathrm{C}$ & Fabaceae \\
\hline 57 & Coccinia grandis (L.) Voigt & $\mathrm{C}$ & Cucurbitaceae \\
\hline 58 & Cocculus hirsutus (L.) W.Theob. & $\mathrm{C}$ & Menispermaceae \\
\hline 59 & Combretum albidum G. Don. & $\mathrm{C}$ & Combretaceae \\
\hline 60 & Commelina attenuata J. König ex Vahl & $\mathrm{H}$ & Commelinaceae \\
\hline 61 & Commelina benghalensis $\mathrm{L}$. & $\mathrm{H}$ & Commelinaceae \\
\hline 62 & Commiphora caudata (Wight \& Arn.) Engl. & $\mathrm{T}$ & Burseraceae \\
\hline 63 & Corallocarpus epigaeus (Rottler) Hook.f. & $\mathrm{C}$ & Cucurbitaceae \\
\hline 64 & Corchorus aestuans L. & $\mathrm{H}$ & Malvaceae \\
\hline 65 & Corypha macropoda Kurz ex Kiden & $\mathrm{T}$ & Arecaceae \\
\hline 66 & Cratavea manga(Lour.) DC. & $\mathrm{T}$ & Capparaceae \\
\hline 67 & Crossandra infundibuliformis (L.) Nees & $\mathrm{H}$ & Acanthaceae \\
\hline 68 & Croton bonplandianus Baill. & $\mathrm{H}$ & Euphorbiaceae \\
\hline 69 & Cyanotis cristata (L.) D.Don & $\mathrm{H}$ & Commelinaceae \\
\hline 70 & Cyanthillium cinereum (L.) H.Rob & $\mathrm{H}$ & Asteraceae \\
\hline 71 & Cynodon dactylon (L.) Pers. & $\mathrm{H}$ & Poaceae \\
\hline 72 & Dalbergia latifolia Roxb. & $\mathrm{T}$ & Fabaceae \\
\hline 73 & Datura metel L. & $\mathrm{H}$ & Solanaceae \\
\hline 74 & Delonix elata (L.) Gamble & $\mathrm{T}$ & Caesalpiniaceae \\
\hline 75 & Dendrophthoe falcata (L.f.) Ettingsh. & $\mathrm{P}$ & Loranthaceae \\
\hline 76 & Derris ovalifolia (Wight \& Arn.) Benth. & $\mathrm{C}$ & Fabaceae \\
\hline 77 & Derris scandens (Roxb.) Benth. & $\mathrm{C}$ & Fabaceae \\
\hline 78 & Desmodium triflorum (L.) DC. & $\mathrm{H}$ & Fabaceae \\
\hline 79 & Dichrostachys cinerea (L.) Wight \& Arn. & $\mathrm{T}$ & Mimosaceae \\
\hline 80 & Dioscorea pentaphylla $\mathrm{L}$. & $\mathrm{C}$ & Dioscoreaceae \\
\hline 81 & Diospyros ebenum J. Koeng. Ex Retz. & $\mathrm{T}$ & Ebenaceae \\
\hline 82 & Diospyros montana Roxb. & $\mathrm{T}$ & Ebenaceae \\
\hline 83 & Diplocyclos palmatus (L.) C.Jeffrey & $\mathrm{C}$ & Cucurbitaceae \\
\hline 84 & Ecbolium viride (Forssk.) Alston & $\mathrm{H}$ & Acanthaceae \\
\hline 85 & Ehretia microphylla Lam. & $\mathrm{S}$ & Boraginaceae \\
\hline 86 & Eucalyptus tereticornis $\mathrm{Sm}$. & $\mathrm{T}$ & Myrtaceae \\
\hline 87 & Euphorbia trigona Mill. & $\mathrm{T}$ & Euphorbiaceae \\
\hline 88 & Evolvulus alsinoides (L.) L. & $\mathrm{H}$ & Convolvulaceae \\
\hline 89 & Evolvulus nummularius (L.) L. & $\mathrm{H}$ & Convolvulaceae \\
\hline 90 & Ficus religiosa $\mathrm{L}$. & $\mathrm{T}$ & Moraceae \\
\hline 91 & Ficus amplissima $\mathrm{Sm}$. & $\mathrm{T}$ & Moraceae \\
\hline 92 & Ficus benghalensis $\mathrm{L}$. & $\mathrm{T}$ & Moraceae \\
\hline 93 & Flacourtia indica (Burm. f.) Merr. & $\mathrm{S}$ & Flacourtiaceae \\
\hline 94 & Flueggea leucopyrus Willd. & $\mathrm{T}$ & Euphorbiaceae \\
\hline 95 & Glinus oppositifolius (L.) Aug.DC. & $\mathrm{H}$ & Molluginaceae \\
\hline 96 & Gloriosa superba L. & $\mathrm{C}$ & Liliaceae \\
\hline 97 & Glycosmis mauritiana (Lam.) Tanaka & $\mathrm{S}$ & Rutaceae \\
\hline 98 & Gmelina arborea Roxb. & $\mathrm{T}$ & Verbenaceae \\
\hline 99 & Gmelina asiatica $\mathrm{L}$. & $\mathrm{C}$ & Verbenaceae \\
\hline 100 & Gymnema sylvestre (Retz.) Schult. & $\mathrm{C}$ & Apocynaceae \\
\hline 101 & Gyrocarpus americanus Jacq. & $\mathrm{T}$ & Hernandiaceae \\
\hline 102 & Hemidesmus indicus (L.) R. Br. ex Schult. & $\mathrm{C}$ & Asclepiadaceae \\
\hline 105 & Hibiscus rosa-sinensis $\mathrm{L}$. & $\mathrm{T}$ & Malvaceae \\
\hline 103 & Hugonia mystax $\mathrm{L}$. & $\mathrm{C}$ & Linaceae \\
\hline 104 & Hybanthus enneaspermus (L.) F.Muell. & $\mathrm{H}$ & Violaceae \\
\hline 106 & Ichnocarpus frutescens (L.) W.T.Aiton & $\mathrm{C}$ & Apocynaceae \\
\hline 107 & Ipomea staphylina Roem. \& Schult. & $\mathrm{C}$ & Convolvulaceae \\
\hline 108 & Jasminum angustifolium (L.) Willd. & $\mathrm{C}$ & Oleaceae \\
\hline 109 & Jasminum auriculatum Vahl & $\mathrm{C}$ & Oleaceae \\
\hline 110 & Jatropha gossypiifolia L. & $\mathrm{S}$ & Euphorbiaceae \\
\hline 111 & Justicia prostrata Gamble & $\mathrm{H}$ & Acanthaceae \\
\hline
\end{tabular}




\begin{tabular}{|c|c|c|c|}
\hline S. No. & Botanical name & Habit & Family \\
\hline 112 & Lantana camara L. & $\mathrm{S}$ & Verbenaceae \\
\hline 113 & Lepisanthes tetraphylla (Vahl) Radlk. & $\mathrm{T}$ & Sapindaceae \\
\hline 114 & Madhuca longifolia (L.) J. F. Macbr. & $\mathrm{T}$ & Sapotaceae \\
\hline 115 & Malvastrum coromandelianum (L.) Garcke & $\mathrm{H}$ & Malvaceae \\
\hline 116 & Mangifera indica $\mathrm{L}$. & $\mathrm{T}$ & Anacardiaceae \\
\hline 117 & Marsdenia brunoniana Wight \& Arn. & $\mathrm{C}$ & Asclepiadaceae \\
\hline 118 & Maytenus emarginata (Ruiz \& Pav.) Loes. & $\mathrm{S}$ & Celastraceae \\
\hline 119 & Mimosa pudica L. & $\mathrm{H}$ & Mimosaceae \\
\hline 120 & Mitragyna parvifolia (Roxb.) Korth. & $\mathrm{T}$ & Rubiaceae \\
\hline 121 & Mollugo pentaphylla $\mathrm{L}$. & $\mathrm{H}$ & Molluginaceae \\
\hline 122 & Morinda pubescens J.E. Smith & $\mathrm{T}$ & Rubiaceae \\
\hline 123 & Moringa oleifera Lam. & $\mathrm{T}$ & Moringaceae \\
\hline 124 & Mukia maderaspatana (L.) M.Roem. & $\mathrm{C}$ & Cucurbitaceae \\
\hline 125 & Nelumbo nucifera Gaertn. & $\mathrm{H}$ & Nelumboginaceae \\
\hline 126 & Nerium oleander $\mathrm{L}$. & $\mathrm{S}$ & Apocynaceae \\
\hline 127 & Nyctanthes arbor-tristis $\mathrm{L}$. & $\mathrm{T}$ & Oleaceae \\
\hline 128 & Ocimum tenuiflorum L. & $\mathrm{H}$ & Lamiaceae \\
\hline 129 & Ocimum americanum $\mathrm{L}$. & $\mathrm{H}$ & Lamiaceae \\
\hline 130 & Oldenlandia umbellata $\mathrm{L}$. & $\mathrm{H}$ & Rubiaceae \\
\hline 131 & Opuntia dillenii (Ker Gawl.) Haw. & $\mathrm{S}$ & Cactaceae \\
\hline 132 & Pachygone ovata (Poir.) Hook. f. \& Thomson & $\mathrm{C}$ & Menispermaceae \\
\hline 133 & Pamburus missionis (Wall. ex Wight) Swingle & $\mathrm{T}$ & Rutaceae \\
\hline 134 & Pandanus odoratissimus L.f. & $\mathrm{T}$ & Pandanaceae \\
\hline 135 & Parthenium hysterophorus L. & $\mathrm{H}$ & Asteraceae \\
\hline 136 & Passiflora foetida $\mathrm{L}$. & $\mathrm{C}$ & Passifloraceae \\
\hline 137 & Pavonia zeylanica (L.) Cav. & $\mathrm{H}$ & Malvaceae \\
\hline 138 & Pedalium murex L. & $\mathrm{H}$ & Pedaliaceae \\
\hline 139 & Peltophorum pterocarpum (DC.) K.Heyne & $\mathrm{T}$ & Caesalpiniaceae \\
\hline 140 & Pentanema indicum $(\mathrm{L}$.$) Ling$ & $\mathrm{H}$ & Asteraceae \\
\hline 141 & Phoenix pusilla Gaertn. & $\mathrm{S}$ & Arecaceae \\
\hline 142 & Phyllanthus amarus Schumach. \& Thonn. & $\mathrm{H}$ & Euphorbiaceae \\
\hline 143 & Physalis minima $\mathrm{L}$. & $\mathrm{H}$ & Solanaceae \\
\hline 144 & Pithecellobium dulce (Roxb.) Benth. & $\mathrm{T}$ & Mimosaceae \\
\hline 145 & Plumbago zeylanica L. & $\mathrm{H}$ & Plumbaginaceae \\
\hline 146 & Pongamia pinnata (L.) Pier. & $\mathrm{T}$ & Fabaceae \\
\hline 147 & Prosopis juliflora (Sw.) Dc. & $\mathrm{T}$ & Mimosaceae \\
\hline 148 & Pterolobium hexapetalum (Roth) Santapau \& Wagh & $\mathrm{C}$ & Caesalpiniaceae \\
\hline 149 & Rivea hypocrateriformis Choisy & $\mathrm{C}$ & Convolvulaceae \\
\hline 150 & Ruellia prostrata Poir. & $\mathrm{H}$ & Acanthaceae \\
\hline 151 & Salvadora persica $\mathrm{L}$. & $\mathrm{T}$ & Salvadoraceae \\
\hline 152 & Sansevieria roxburghiana Schult. \& Schult. f. & $\mathrm{H}$ & Asparagaceae \\
\hline 153 & Santalum album L. & $\mathrm{T}$ & Santalaceae \\
\hline 154 & Sarcostemma acidum (Roxb.) Voigt & $\mathrm{C}$ & Asclepiadaceae \\
\hline 155 & Scoparia dulcis $\mathrm{L}$. & $\mathrm{H}$ & Scrophulariaceae \\
\hline 156 & Scutia myrtina (Burm. f.) Kurz & $\mathrm{C}$ & Rhamnaceae \\
\hline 157 & Secamone emetica (Retz.) R. Br. ex Schult. & $\mathrm{C}$ & Apocynaceae \\
\hline 158 & Senna auriculata (L.) Roxb. & $\mathrm{S}$ & Caesalpiniaceae \\
\hline 159 & Senna occidentalis (L.) Link & $\mathrm{S}$ & Caesalpiniaceae \\
\hline 160 & Senna tora (L.) Roxb. & S & Caesalpiniaceae \\
\hline 161 & Senna siamea (Lam.) H. S. Irwin \& Barneby & $\mathrm{T}$ & Mimosaceae \\
\hline 162 & Sida acuta Burm.f. & $\mathrm{H}$ & Malvaceae \\
\hline 163 & Sida cordifolia $\mathrm{L}$. & $\mathrm{H}$ & Malvaceae \\
\hline 164 & Solanum trilobatum $\mathrm{L}$. & $\mathrm{C}$ & Solanaceae \\
\hline 165 & Solanum xanthocarpum Schrad. \& H. Wendl. & $\mathrm{H}$ & Solanaceae \\
\hline 166 & Spermacoce hispida $\mathrm{L}$. & $\mathrm{H}$ & Rubiaceae \\
\hline 167 & Stachytarpheta jamaicensis auct.non(L.) Vahl & $\mathrm{H}$ & Verbenaceae \\
\hline
\end{tabular}




\begin{tabular}{|c|c|c|c|}
\hline S. No. & Botanical name & Habit & Family \\
\hline 168 & Streblus asper Lour. & $\mathrm{T}$ & Moraceae \\
\hline 169 & Strychnos nux-vomica L. & $\mathrm{T}$ & Loganiaceae \\
\hline 170 & Strychnos potatorum L. f. & $\mathrm{T}$ & Loganiaceae \\
\hline 171 & Syzygium cumini (L.) Skeels & $\mathrm{T}$ & Myrtaceae \\
\hline 172 & Tabernaemontana divaricata (L.) R.Br. ex Roem. \& Schult. & $\mathrm{S}$ & Apocynaceae \\
\hline 173 & Tagetus erecta $\mathrm{L}$. & $\mathrm{H}$ & Asteraceae \\
\hline 174 & Tamarindus indica $\mathrm{L}$. & $\mathrm{T}$ & Caesalpiniaceae \\
\hline 175 & Tarenna asiatica (L.) Kuntze ex K.Schum. & $\mathrm{S}$ & Rubiaceae \\
\hline 176 & Tecoma stans (L.) Juss. ex Kunth & $\mathrm{T}$ & Bignoniaceae \\
\hline 177 & Tectona grandis L.f. & $\mathrm{T}$ & Verbenaceae \\
\hline 178 & Tephrosia purpurea (L.) Pers. & $\mathrm{H}$ & Fabaceae \\
\hline 179 & Thespesia populnea (L.) Sol. ex Corrêa & $\mathrm{T}$ & Malvaceae \\
\hline 180 & Tinospora cordifolia (Willd.) Hook. f. \& Thomson & $\mathrm{C}$ & Menispermaceae \\
\hline 181 & Toddalia asiatica (L.) Lam. & $\mathrm{C}$ & Rutaceae \\
\hline 182 & Tribulus terrestris L. & $\mathrm{H}$ & Zygophyllaceae \\
\hline 183 & Tridax procumbens $\mathrm{L}$. & $\mathrm{H}$ & Asteraceae \\
\hline 184 & Tylophora indica (Burm. f.) Merr. & $\mathrm{C}$ & Asclepiadaceae \\
\hline 185 & Ventilago maderaspatana Gaertn. & $\mathrm{C}$ & Rhamnaceae \\
\hline 186 & Vitex negundo L. & $\mathrm{T}$ & Laminaceae \\
\hline 187 & Wrightia tinctoria (Roxb.) R. Br & $\mathrm{T}$ & Apocynaceae \\
\hline 188 & Xanthium indicum J.Koenig & $\mathrm{H}$ & Asteraceae \\
\hline 189 & Ziziphus mauritiana Lam. & $\mathrm{T}$ & Rhamnaceae \\
\hline 190 & Ziziphus oenoplia (L.) Mill. & $\mathrm{C}$ & Rhamnaceae \\
\hline
\end{tabular}

C-Climber; H - Herb; S - Shrub; T - Tree; P - Parasite.

Table 2. List of Plants recorded in the Vadakkanandal sacred grove.

\begin{tabular}{|c|c|c|c|}
\hline S. No. & Botanical name & Habit & Family \\
\hline 1 & Abrus precatorius L. & $\mathrm{C}$ & Fabaceae \\
\hline 2 & Abutilon indicum (L.) Sweet & $\mathrm{H}$ & Malvaceae \\
\hline 3 & Acacia ferruginea DC. & $\mathrm{T}$ & Mimosaceae \\
\hline 4 & Acacia leucophloea (Roxb.) Willd. & $\mathrm{T}$ & Mimosaceae \\
\hline 5 & Acacia planifrons Wight \& Arn. & $\mathrm{T}$ & Mimosaceae \\
\hline 6 & Acacia caesia (L.) Willd. & $\mathrm{C}$ & Mimosaceae \\
\hline 7 & Acacia nilotica (L.) Delile & $\mathrm{T}$ & Mimosaceae \\
\hline 8 & Acalypha indica $\mathrm{L}$. & $\mathrm{H}$ & Euphorbiaceae \\
\hline 9 & Achyranthes aspera $\mathrm{L}$. & $\mathrm{H}$ & Amaranthaceae \\
\hline 10 & Aerva lanata (L.) Juss. ex Schult. & $\mathrm{H}$ & Amaranthaceae \\
\hline 11 & Alangium salviifolium (L. f.) Wangerin & $\mathrm{T}$ & Alangiaceae \\
\hline 12 & Albizia amara (Roxb.) Boivin & $\mathrm{T}$ & Mimosaceae \\
\hline 13 & Albizia lebbeck (L.) Benth. & $\mathrm{T}$ & Mimosaceae \\
\hline 14 & Allophylus serratus (Roxb.) kurz. & $\mathrm{S}$ & Sapindaceae \\
\hline 15 & Aloe vera (L.) Burm.f. & $\mathrm{H}$ & Asphodelaceae \\
\hline 16 & Amarantus viridis $\mathrm{L}$. & $\mathrm{H}$ & Amaranthaceae \\
\hline 17 & Andrographis paniculata (Burm.f.) Nees & $\mathrm{H}$ & Acanthaceae \\
\hline 18 & Annona squamosa $\mathrm{L}$. & $\mathrm{T}$ & Annonaceae \\
\hline 19 & Apluda mutica L. & $\mathrm{H}$ & Poaceae \\
\hline 20 & Aristolochia indica L. & $\mathrm{C}$ & Aristolochiaceae \\
\hline 21 & Asparagus racemosus Willd. & $\mathrm{C}$ & Asparagaceae \\
\hline 22 & Atalantia monophylla (L.) DC. & $\mathrm{T}$ & Rutaceae \\
\hline 23 & Azadirachta indca A. Juss. & $\mathrm{T}$ & Meliaceae \\
\hline 24 & Azima tetracantha Lam. & $\mathrm{S}$ & Salvadoraceae \\
\hline 25 & Bambusa bambos (L.) Voss & $\mathrm{S}$ & Poaceae \\
\hline 26 & Barleria prionitis L. & $\mathrm{H}$ & Acanthaceae \\
\hline 27 & Basilicum polystachyon (L.) Moench & $\mathrm{H}$ & Laminaceae \\
\hline 28 & Blepharis maderaspatensis (L.) B.Heyne ex Roth & $\mathrm{H}$ & Acanthaceae \\
\hline
\end{tabular}




\begin{tabular}{|c|c|c|c|}
\hline S. No. & Botanical name & Habit & Family \\
\hline 29 & Boerhavia diffusa $\mathrm{L}$. & $\mathrm{H}$ & Nyctaginaceae \\
\hline 30 & Borassus flabellifer $\mathrm{L}$. & $\mathrm{T}$ & Arecaceae \\
\hline 31 & Bougainvillaea spectabilis, Willd. & $\mathrm{S}$ & Nyctaginaceae \\
\hline 32 & Bulbostylis barbata (Rottb.) Kunth ex C.B. Clarke & $\mathrm{H}$ & Cyperaceae \\
\hline 33 & Butea monosperma (Lam.) Taub. & $\mathrm{T}$ & Fabaceae \\
\hline 34 & Cadaba fruticosa (L.) Druce & $\mathrm{S}$ & Capparaceae \\
\hline 35 & Caesalpinia bonduc (L.) Roxb. & $\mathrm{C}$ & Caesalpiniaceae \\
\hline 36 & Calotropis gigantea (L.) Dryand. & $\mathrm{S}$ & Asclepiadaceae \\
\hline 37 & Calycopteris floribunda Lam. & $\mathrm{C}$ & Combretaceae \\
\hline 38 & Canavalia virosa (Roxb.) & $\mathrm{C}$ & Fabaceae \\
\hline 39 & Canthium coromandelicum (Burm.f.) Alston & $\mathrm{S}$ & Rubiaceae \\
\hline 40 & Capparis brevispina DC. & $\mathrm{C}$ & Capparaceae \\
\hline 41 & Capparis sepiaria $\mathrm{L}$. & $\mathrm{C}$ & Capparaceae \\
\hline 42 & Capparis zeylanica $\mathrm{L}$. & $\mathrm{C}$ & Capparaceae \\
\hline 43 & Cardiospermum helicacabum L. & $\mathrm{C}$ & Sapindaceae \\
\hline 44 & Carissa spinarum $\mathrm{L}$. & $\mathrm{S}$ & Apocynaceae \\
\hline 45 & Carmona retusa (Vahl) Masam. & $\mathrm{S}$ & Boraginaceae \\
\hline 46 & Cascabela thevitia (L.) Lipp. & $\mathrm{T}$ & Apocynaceae \\
\hline 47 & Cassia fistula $\mathrm{L}$. & $\mathrm{T}$ & Caesalpiniaceae \\
\hline 48 & Cassine glauca (Rottb.) Kuntze & $\mathrm{T}$ & Celastraceae \\
\hline 49 & Cassytha filiformis L. & $\mathrm{P}$ & Lauraceae \\
\hline 50 & Casuarina equisetifolia L. & $\mathrm{T}$ & Casuarinaceae \\
\hline 51 & Catharanthus roseus (L.) G.Don & $\mathrm{H}$ & Apocynaceae \\
\hline 52 & Catunaregam spinosa (Thunb.) Tirven. & $\mathrm{S}$ & Rubiaceae \\
\hline 53 & Chloris barbata $\mathrm{Sw}$. & $\mathrm{H}$ & Poaceae \\
\hline 54 & Chloroxylon swietenia DC. & $\mathrm{T}$ & Rutaceae \\
\hline 55 & Cissampelos pareira L. var. hirsuta (Buch.-Ham. ex DC.) Forman & $\mathrm{C}$ & Menispermaceae \\
\hline 56 & Cissus quadrangularis $\mathrm{L}$. & $\mathrm{C}$ & Vitaceae \\
\hline 57 & Cleome gynandra $\mathrm{L}$. & $\mathrm{H}$ & Capparaceae \\
\hline 58 & Cleome viscosa $\mathrm{L}$. & $\mathrm{H}$ & Capparaceae \\
\hline 59 & Clitoria ternatea $\mathrm{L}$. & $\mathrm{C}$ & Fabaceae \\
\hline 60 & Coccinia grandis (L.) Voigt & $\mathrm{C}$ & Cucurbitaceae \\
\hline 61 & Cocculus hirsutus (L.) W.Theob. & $\mathrm{C}$ & Menispermaceae \\
\hline 62 & Combretum albidum G. Don. & $\mathrm{C}$ & Fabaceae \\
\hline 63 & Convolvulus arvensis $\mathrm{L}$. & $\mathrm{H}$ & Convolvulaceae \\
\hline 64 & Corchorus aestuans $\mathrm{L}$. & $\mathrm{H}$ & Malvaceae \\
\hline 65 & Cordia monoica Roxb. & $\mathrm{T}$ & Boraginaceae \\
\hline 66 & Cratavea manga(Lour.) DC. & $\mathrm{T}$ & Capparaceae \\
\hline 67 & Crossandra infundibuliformis (L.) Nees & $\mathrm{H}$ & Acanthaceae \\
\hline 68 & Croton bonplandianus Baill. & $\mathrm{H}$ & Euphorbiaceae \\
\hline 69 & Cucumis maderaspatana $\mathrm{L}$. & $\mathrm{C}$ & Cucurbitaceae \\
\hline 70 & Cyanotis cristata (L.) D. Don & $\mathrm{H}$ & Commelinaceae \\
\hline 71 & Cyanthillium cinereum (L.) H.Rob & $\mathrm{H}$ & Asteraceae \\
\hline 72 & Cyperus rotundus $\mathrm{L}$. & $\mathrm{H}$ & Cyperaceae \\
\hline 73 & Dalbergia sissoo Roxb. ex DC. & $\mathrm{C}$ & Fabaceae \\
\hline 74 & Datura metel L. & $\mathrm{H}$ & Solanaceae \\
\hline 75 & Delonix elata (L.) Gamble & $\mathrm{T}$ & Caesalpiniaceae \\
\hline 76 & Dendrophthoe falcata (L.f.) Ettingsh. & $\mathrm{P}$ & Loranthaceae \\
\hline 77 & Derris ovalifolia (Wight \& Arn.) Benth. & $\mathrm{C}$ & Fabaceae \\
\hline 78 & Derris scandens (Roxb.) Benth. & $\mathrm{C}$ & Fabaceae \\
\hline 79 & Dichrostachys cinerea (L.) Wight \& Arn. & $\mathrm{T}$ & Mimosaceae \\
\hline 80 & Dioscorea pentaphylla $\mathrm{L}$. & $\mathrm{C}$ & Dioscoreaceae \\
\hline 81 & Dioscorea oppositifolia L. & $\mathrm{C}$ & Dioscoreaceae \\
\hline 82 & Diospyros ebenum J. Koeng. Ex Retz. & $\mathrm{T}$ & Ebenaceae \\
\hline 83 & Euphorbia antiquorum L. & $\mathrm{T}$ & Euphorbiaceae \\
\hline 84 & Evolvulus alsinoides L. & $\mathrm{H}$ & Convolvulaceae \\
\hline
\end{tabular}




\begin{tabular}{|c|c|c|c|}
\hline S. No. & Botanical name & Habit & Family \\
\hline 85 & Evolvulus nummularis L. & $\mathrm{H}$ & Convolvulaceae \\
\hline 86 & Ficus amplissima J. E. & $\mathrm{T}$ & Moraceae \\
\hline 87 & Ficus religiosa $\mathrm{L}$. & $\mathrm{T}$ & Moraceae \\
\hline 88 & Ficus benghalensis L. & $\mathrm{T}$ & Moraceae \\
\hline 89 & Ficus hispida L. f. & $\mathrm{T}$ & Moraceae \\
\hline 90 & Flacourtia indica (Burm. f.) Merr. & $\mathrm{S}$ & Flacourtiaceae \\
\hline 91 & Flueggea leucopyrus Willd. & $\mathrm{S}$ & Euphorbiaceae \\
\hline 92 & Glinus oppositifolius (L.) Aug.DC. & $\mathrm{H}$ & Molluginaceae \\
\hline 93 & Glycosmis mauritiana (Lam.) Tanaka & $\mathrm{S}$ & Rutaceae \\
\hline 94 & Gmelina asiatica $\mathrm{L}$. & $\mathrm{C}$ & Verbenaceae \\
\hline 95 & Grewia orientalis L. & $\mathrm{C}$ & Tiliaceae \\
\hline 96 & Gymnema sylvestre (Retz.) Schult. & $\mathrm{C}$ & Apocynaceae \\
\hline 97 & Gyrocarpus americanus Jacq. & $\mathrm{T}$ & Hernandiaceae \\
\hline 98 & Hemidesmus indicus (L.) R. Br. ex Schult. & $\mathrm{H}$ & Asclepiadaceae \\
\hline 99 & Hybanthus enneaspermus (L.) F.v. Muell. & $\mathrm{H}$ & Violaceae \\
\hline 100 & Hygrophila auriculata (K.Schum.)Heine & $\mathrm{H}$ & Acanthaceae \\
\hline 101 & Ichnocarpus frutescens (L.) W.T.Aiton & $\mathrm{C}$ & Apocynaceae \\
\hline 102 & Ipomoea carnea Jacq. & $\mathrm{S}$ & Convolvulaceae \\
\hline 103 & Jasminum angustifolium (L.) Willd. & $\mathrm{C}$ & Oleaceae \\
\hline 104 & Jatropha gossypiifolia L. & $\mathrm{S}$ & Euphorbiaceae \\
\hline 105 & Lantana camara $\mathrm{L}$. & $\mathrm{S}$ & Verbinaceae \\
\hline 106 & Lepisanthes tetraphylla (Vahl) Radlk. & $\mathrm{T}$ & Sapindaceae \\
\hline 107 & Limonia acidissima $\mathrm{L}$. & $\mathrm{T}$ & Rutaceae \\
\hline 108 & Madhuca longifolia (L.) J. F. Macbr. & $\mathrm{T}$ & Sapotaceae \\
\hline 109 & Malvastrum coromandelianum (L.) Garcke & $\mathrm{H}$ & Malvaceae \\
\hline 110 & Mangifera indica $\mathrm{L}$. & $\mathrm{T}$ & Anacardiaceae \\
\hline 111 & Maytenus emarginata (Ruiz \& Pav.) Loes. & $\mathrm{S}$ & Celastraceae \\
\hline 112 & Merremia tridentata (L.) Hall.f. & $\mathrm{H}$ & Convolvulaceae \\
\hline 113 & Mimosa pudica L. & $\mathrm{H}$ & Mimosaceae \\
\hline 114 & Mitragyna parvifolia (Roxb.) Korth. & $\mathrm{T}$ & Rubiaceae \\
\hline 115 & Mollugo pentaphylla L. & $\mathrm{H}$ & Molluginaceae \\
\hline 116 & Morinda pubescens J.E. Smith & $\mathrm{T}$ & Rubiaceae \\
\hline 117 & Moringa oleifera Lam. & $\mathrm{T}$ & Moringaceae \\
\hline 118 & Mukia maderaspatana (L.) M.Roem. & $\mathrm{C}$ & Cucurbitaceae \\
\hline 119 & Nerium oleander L. & $\mathrm{S}$ & Apocynaceae \\
\hline 120 & Nyctanthes arbor-tristis L. & $\mathrm{T}$ & Oleaceae \\
\hline 121 & Ocimum cannum Sims L. & $\mathrm{H}$ & Lamiaceae \\
\hline 122 & Ocimum tenuiflorum $\mathrm{L}$. & $\mathrm{H}$ & Lamiaceae \\
\hline 123 & Oldenlandia umbellata $\mathrm{L}$. & $\mathrm{H}$ & Rubiaceae \\
\hline 124 & Opuntia dillenii (Ker Gawl.) Haw. & $\mathrm{H}$ & Cactaceae \\
\hline 125 & Parthenium hysterophorus L. & $\mathrm{H}$ & Asteraceae \\
\hline 126 & Passiflora foetida $\mathrm{L}$. & $\mathrm{C}$ & Passifloraceae \\
\hline 127 & Pavonia zeylanica & $\mathrm{H}$ & Malvaceae \\
\hline 128 & Pedalium murex L. & $\mathrm{H}$ & Pedaliaceae \\
\hline 129 & Peltophorum pterocarpum (DC.) K.Heyne & $\mathrm{T}$ & Caesalpiniaceae \\
\hline 130 & Phoenix pusilla Gaertn. & $\mathrm{S}$ & Arecaceae \\
\hline 131 & Phyllanthus amarus Schumach. \& Thonn. & $\mathrm{H}$ & Euphorbiaceae \\
\hline 132 & Physalis minima $\mathrm{L}$. & $\mathrm{H}$ & Solanaceae \\
\hline 133 & Pithecellobium dulce (Roxb.) Benth. & $\mathrm{T}$ & Mimosaceae \\
\hline 134 & Pleiospermium alatum (Wight \& Arn.) Swingle & $\mathrm{T}$ & Rutaceae \\
\hline 135 & Plumbago zeylanica L. & $\mathrm{H}$ & Plumbaginaceae \\
\hline 136 & Pongamia pinnata (L.) Pier. & $\mathrm{T}$ & Fabaceae \\
\hline 137 & Prosopis juliflora (sw.) Dc. & $\mathrm{T}$ & Mimosaceae \\
\hline 138 & Pterolobium hexapetalum (Roth) Santapau \& Wagh & $\mathrm{C}$ & Caesalpiniaceae \\
\hline 139 & Reissantia indica (Willd.) N. Hallé & $\mathrm{C}$ & Celastraceae \\
\hline 140 & Rivea hypocrateriformis Choisy & $\mathrm{C}$ & Convolvulaceae \\
\hline
\end{tabular}




\begin{tabular}{|c|c|c|c|}
\hline S. No. & Botanical name & Habit & Family \\
\hline 141 & Ruellia prostrata Poir. & $\mathrm{H}$ & Acanthaceae \\
\hline 142 & Salvadora persica $\mathrm{L}$. & $\mathrm{T}$ & Salvadoraceae \\
\hline 143 & Sansevieria roxburghiana Schult. \& Schult. f. & $\mathrm{H}$ & Asparagaceae \\
\hline 144 & Sapindus emarginatus Vahl & $\mathrm{T}$ & Sapindaceae \\
\hline 145 & Sarcostemma acidum (Roxb.) Voigt & $\mathrm{C}$ & Asclepiadaceae \\
\hline 146 & Scoparia dulcis $\mathrm{L}$. & $\mathrm{H}$ & Scrophulariaceae \\
\hline 147 & Scutia myrtina (Burm. f.) Kurz & $\mathrm{C}$ & Rhamnaceae \\
\hline 148 & Secamone emetica (Retz.) R. Br. ex Schult. & $\mathrm{C}$ & Apocynaceae \\
\hline 149 & Senna auriculata (L.) Roxb. & $\mathrm{S}$ & Caesalpiniaceae \\
\hline 150 & Senna occidentalis (L.) Link & $\mathrm{S}$ & Caesalpiniaceae \\
\hline 151 & Senna siamea (Lam.) H. S. Irwin \& Barneby & $\mathrm{T}$ & Mimosaceae \\
\hline 152 & Sida acuta Burm.f. & $\mathrm{H}$ & Malvaceae \\
\hline 153 & Solanum triflorum Nutt. & $\mathrm{H}$ & Solanaceae \\
\hline 154 & Spermacoce hispida L. & $\mathrm{H}$ & Rubiaceae \\
\hline 155 & Streblus asper Lour. & $\mathrm{T}$ & Moraceae \\
\hline 156 & Strychnos nux-vomica $\mathrm{L}$. & $\mathrm{T}$ & Loganiaceae \\
\hline 157 & Strychnos potatorum L. f. & $\mathrm{T}$ & Loganiaceae \\
\hline 158 & Syzygium cumini (L.) Skeels & $\mathrm{T}$ & Myrtaceae \\
\hline 159 & Tabernaemontana divaricata (L.) R.Br. ex Roem. \& Schult. & $\mathrm{S}$ & Apocynaceae \\
\hline 160 & Tamarindus indica $\mathrm{L}$. & $\mathrm{T}$ & Caesalpiniaceae \\
\hline 161 & Tarenna asiatica (L.) Kuntze ex K.Schum. & $\mathrm{S}$ & Rubiaceae \\
\hline 162 & Tecoma stans (L.) Juss. ex Kunth & $\mathrm{T}$ & Bignoniaceae \\
\hline 163 & Tectona grandis L.f. & $\mathrm{T}$ & Verbenaceae \\
\hline 164 & Tephrosia purpurea (L.) pers. & $\mathrm{H}$ & Fabaceae \\
\hline 165 & Terminalia arjuna (Roxb.ex DC.) Wt.\& Arn & $\mathrm{T}$ & Combretaceae \\
\hline 166 & Theriophonum minutum (Willd.) Baill. & $\mathrm{H}$ & Araceae \\
\hline 167 & Thespesia populnea (L.) Sol. ex Corrêa & $\mathrm{T}$ & Malvaceae \\
\hline 168 & Tinospora cordifolia (Willd.) Hook. f. \& Thomson & $\mathrm{C}$ & Menispermaceae \\
\hline 169 & Toddalia asiatica (L.) Lam. & $\mathrm{C}$ & Rutaceae \\
\hline 170 & Tribulus terrestris $\mathrm{L}$. & $\mathrm{H}$ & Zygophyllaceae \\
\hline 171 & Tridax procumbens $\mathrm{L}$. & $\mathrm{H}$ & Asteraceae \\
\hline 172 & Triumfetta rhomboidea Jacq. & $\mathrm{H}$ & Tiliaceae \\
\hline 173 & Tylophora indica (Burm. f.) Merr. & $\mathrm{C}$ & Asclepiadaceae \\
\hline 174 & Ventilago maderaspatana Gaertn. & $\mathrm{C}$ & Rhamnaceae \\
\hline 175 & Vitex negundo L. & $\mathrm{T}$ & Laminaceae \\
\hline 176 & Wrightia tinctoria (Roxb.) R. Br & $\mathrm{T}$ & Apocynaceae \\
\hline 177 & Ziziphus mauritiana Lam. & $\mathrm{T}$ & Rhamnaceae \\
\hline 178 & Ziziphus oenoplia (L.) Mill. & $\mathrm{C}$ & Rhamnaceae \\
\hline
\end{tabular}

C-Climber; H - Herb; S - Shrub; T - Tree; P - Parasite.



Fig. 4: Dominant habits in Palrampattu sacred grove.

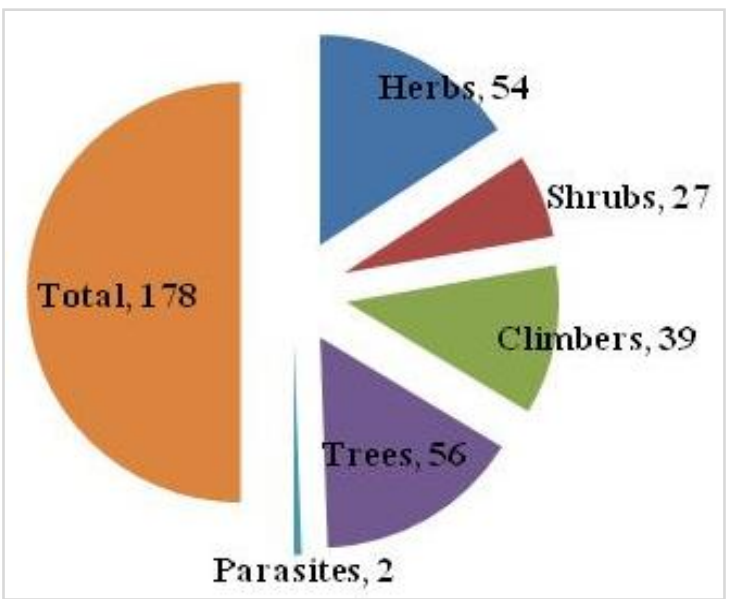

Fig. 5: Dominant habits in Vadakanandal sacred grove. 


\section{Soliamman grove at Palrampattu}

It is situated in Palarampattu village, Kallakurichi Taluk of Villupuram district. The deities associated with this grove are Aagasa Karuppusami, Periyakruppu and Nondikaruppu. There are also water bodies which are used for agricultural practices. The grove is surrounded by Karadicithoor on the west, Ammapettai on the south, Mathur on the north and Mathvacheri on the east. Pooja is conducted every day and special poojas are performed on Tuesdays and Fridays. The ceremony of ear boring is performed in the month of Aadi (mid- July to mid-August) during the Urani Pongal. Much importance is given to women. Animals such as goat and fowl are sacrificed. The administration is conducted by the village committees. The poojari (the person who is performing poojas) is from the Vanniya caste. During Urani Pongal, the places are cleared and temporary constructions are made. The grazing of cattle around the grove is permitted.

\section{Thiyagapaadi amman grove at Vadakanandal}

Vadakanandal is situated at Kallakurichi taluk of Viluppuram district. Kachirayapalayam, Akkarayapalayam, Mettupalayam, Kamaraj salai are the main places of Vadakananadal. The goddess Thiyagapaadi Amman temple is considered to be one of the powerful goddesses in the surrounding villages. The grove is dedicated to Thiyagapaadi Amman and is surrounded by the Gomukhi dam on the north, Palrampattu village on the south, Kachirayapalyam on the west and Parigam village on the east. Greenery can be seen throughout the year because of the Gomukhi dam. Sugarcane, rice and turmeric are cultivated because of the availability of water. Other deities like Karuppaiya and Putru (ant-hill) Mariamman are also worshipped in this grove. Poojas are performed everyday throughout the year. A car festival is organized once in five years during the month of Aadi (mid-July to mid-August). Coconut milk, curd and navadhanyas are offered to the goddess during the festival. Goats and fowl are sacrificed to the deity Karuppaiya who is also associated with this grove.

\section{Disturbance analysis}

Both the groves are managed by the local village communities. Nowadays, concrete buildings are being constructed by destroying the grove for the benefit of the visitors. The local people use the grove for cattle grazing and also collect fuel wood by cutting small trees. The grove has also become a refuge for drunkards.

\section{Conclusion}

A total number of plants of 190 species belong to 168 genera from the Palrampattu grove. The plants include herbs (59 species), shrubs (28 species), climbers (43 species), trees (58 species), and parasite ( 2 species). In Vadakandhal sacred grove, a total of 178 species belong to 159 genera, herbs 54 species, shrubs 27 species, climbers 39 species, trees 56 species and parasites 2 species.

Sacred groves are thus one of the most valuable sources of plant diversity, ethnobotany, non timber forests products and cultural aspects. The taboos, rituals and beliefs associated with the groves, supported by mystic folklore have been the prime motivating factors for preserving them in a pristine condition. In short, the floristic diversity of the Palrampattu and Vadakanandal sacred groves act as a storehouse of medicinal plants. Many of these plants are valuable to the village communities as well as modern pharmacopoeia. It is also a storehouse of rare, endangered and threatened plants.

\section{Conflict of interest statement}

Authors declare that they have no conflict of interest.

\section{References}

Amairthalingam, M., 2012. Sacred Groves of Tamil Nadu and their Management. Forest Department, Government of Tamil Nadu, Chennai.

Amirthalingam, M., 1998. Sacred Groves of Tamil Nadu, C.P.R. Environment Education Centre, Chennai.

Balakrishnan, N.P., Chakrabarty, T., 2007. The Family Euphorbiaceae in India - A Synopsis of its Profile, Taxonomy and Bibliography. Bishen Singh Mahendra Pal Singh, Dehra Dun. 500p.

Brandis, D., 1897. Indigenous Indian forestry: Sacred groves. In: Indian Forestry. Oriental Institute, Working. pp.12-13.

Fergusson, J.A., 1971. Tree and Serpent Worship. India Book House, Varanasi.

Gadgil, M., Vartak, V. D., 1975. Sacred groves of India - A plea for continued conservation, J. Bombay Nat. Hist. Soc.72, 314-321.

Gadgil, M., Vartak, V. D., 1981. Sacred Groves of Maharashtra: An Inventory. In: Glimpses of Ethnobotany (Jain, S.K.). Oxford University Press, Bombay. pp. 279294.

Gamble, J. S.., Fisher, C. E. C., 1915-1935 and 1965. Flora of the Presidency of Madras, Vol. I-III.

Henry, A. N., Chithra, V., Balakrishnan, N. P., 1989. Flora of Tamil Nadu, India. Series 1: Analysis. Vol. 3. Botanical Survey of India, Coimbatore. 173p. 
Henry, A.N., Kumari, G.R., Chithra, V., 1987. Flora of Tamil Nadu, India. Series 1: Analysis. Vol. 2. Botanical Survey of India, Coimbatore. 258p.

Kadamban, D. 1998. Biocultural Perspective and Plant Diversity of Sacred Groves and Traditional Medical Knowledge in Pondicherry Environs. Ph.D. thesis, Pondicherry University. pp.149.

Karthik, S., Subramanian, M., Ravikumar, S., and Dhamotharan, R., 2015. 'Floristic Studies on Kilcheruvi (Edaicheruvi) Sacred Grove at Cuddalore District, Tamil Nadu, South India'. Int. J. Curr. Res. Biosci. Plant Biol. 2(7), 192-205.

Karthikeyan, S., Sanjappa, M., Moorthy, S., 2009. Flowering Plants of India - Dicotyledons, Vol. 1 (AcanthaceaeAviciniaceae). Botanical Survey of India, Kolkata. 365p.

Maheswaran, B., Dayanandan, P., and Narasimha, D., 1995. Miniature sacred groves near Vedanthangal bird sanctuary. In: Abstracts of 2nd Congress on Traditional Science and Technology of India. Bio.3. December 26-31, Madras.

Malhotra, K.C, Gokhale, Y, Chatterjee, S. and Srivastava, S. 2001. Cultural and Ecological Dimensions of Sacred Groves in India, New Delhi: Indian National Science Academy; Bhopal: Indira Gandhi Rashtriya Manav Sangrahalaya.
Matthew, K.M., 1982. Illustrations on the Flora of the Tamilnadu Carnatic. Vol. 2. The Diocesan Press, Madras. $1027 p$.

Matthew, K.M., 1983. The Flora of the Tamilnadu Carnatic. Vol. 3 (Parts 1 and 2). The Diocesan Press, Madras. $2154 p$.

Matthew, K.M., 1988. Further Illustrations on the Flora of the Tamilnadu Carnatic. Vol. 4. The Diocesan Press, Madras. 915p.

Nair, N.C., Henry, A.N., 1983. Flora of Tamil Nadu, India. Series I: Analysis. Vol. 1. Botanical Survey of India, Coimbatore. 188p.

Ramakrishnan, P. S., Saxena, K. G., Chandrashekara, U. M. (Eds.), 1998. Conserving the Sacred for Biodiversity Management. Oxford and IBH Publishing Co., New Delhi. pp.69-46.

Ramanujam, M. P., Kadamban, D., Kumaravelu, G., Praveenkumar, K., 2002. Sacred Groves - An Overview. In: Ethnobotany. Aavishkar Publishers, Jaipur. pp.13-53.

Sanjappa, M., 1992. Legumes of India. Bishen Singh Mahendra Pal Singh, Dehra Dun. 338p.

Somashekar, B.S. 1998. Treasure House in Trouble. Amruth. 2(5), 3-7.

\section{How to cite this article:}

Subramanian, M., Karthik, S., Ravikumar, S., Dhamodaran, R., 2016. A study on the plant biocultural diversity of Palrampattu and Vadakanandal sacred groves in Villupuram District, Tamil Nadu. Int. J. Curr. Res. Biosci. Plant Biol. 3(6), 92-104. doi: http://dx.doi.org/10.20546/ijcrbp.2016.306.012 